\title{
Docetaxel vs. Novel Hormonal Agent for Upfront Management of De Novo Metastatic Hormone- Sensitive Prostate Cancer: A Comprehensive Report of a Single-Center Experience
}

\section{Sunny Guin}

Sema4

Bobby K. Liaw

Mount Sinai Health System

Tomi Jun

Mount Sinai Health System

Kristin Ayers

Sema4

Bonny Patel

Sema 4

Timmy O'Connell

Sema 4

Matthew Deitz

Sema4

Michael Klein

Sema4

Tommy Mullaney

Sema4

Tony Prentice

Sema 4

Scott Newman

Sema4

Marc Fink

Sema 4

Xiang Zhou

Sema 4

Eric E . Schadt

Sema 4

Rong Chen

Sema4 
William Oh ( $\nabla$ william.oh@sema4.com )

Sema4

\section{Research Article}

Keywords: metastatic hormone sensitive prostate cancer, docetaxel, novel hormonal agents, failure free survival, metastasis burden, PSA prognostic, Sema4 database

Posted Date: July 29th, 2021

DOl: https://doi.org/10.21203/rs.3.rs-659032/v1

License: (c) (1) This work is licensed under a Creative Commons Attribution 4.0 International License. Read Full License 


\section{Abstract}

Background: Upfront docetaxel or novel hormonal agents (NHA) such as abiraterone and enzalutamide have become the standard of care for metastatic hormone sensitive prostate cancer (mHSPC). However, data comparing the efficacy of docetaxel and NHAs in this setting are limited.

Patients and Methods: This was a retrospective cohort study of patients with de novo mHSPC treated with upfront docetaxel or an NHA between January 1, 2014 and April 30, 2019 within the Mount Sinai Health System. Clinical data were extracted from the medical record. The primary outcome was failurefree survival (FFS), defined as the time to next treatment. The primary predictor was treatment with docetaxel or NHA. FFS was compared between the two groups using the Kaplan Meier method and multivariable Cox proportional hazards models. We additionally assessed the prognostic value of posttreatment PSA.

Results: We identified 94 de novo mHSPC patients; 52 and 42 treated with upfront docetaxel and NHAs, respectively. NHAs were associated with significantly longer FFS compared to docetaxel (20.7 vs. 10.1 months, $p=0.023$ ). In a multivariable model adjusting for demographics and clinical factors, docetaxel was independently associated with worse FFS compared to NHAs (HR 1.96, 95\% Cl 1.12-3.45, p=0.019). High metastasis burden patients had a significantly longer FFS with NHAs than docetaxel (25.12 vs. 9.63 months, $p=0.014$ ), while there was no significant difference in FFS among low metastasis burden patients (NHA 20.71 vs. Docetaxel 26.5 months, $p=0.9$ ). Regardless of treatment, lower post-treatment PSA levels were associated with improved FFS (58.95 vs. 11.57 vs. 9.4 months for PSA $\leq 0.2,0.2-0.4$, $>0.4 \mathrm{ng} / \mathrm{ml}$, respectively; $\mathrm{p}<0.001$ )

Conclusion: Comparative analysis of real-world data demonstrated longer FFS in de novo mHSPC treated with NHA compared to docetaxel. In addition, the depth of PSA response following combination treatment may hold prognostic value for mHSPC outcomes.

\section{Background}

The standard of care for metastatic hormone sensitive prostate cancer (mHSPC) continues to quickly evolve. While the use of androgen deprivation therapy (ADT) remains foundational, the earlier incorporation of advanced therapeutic agents previously reserved for castration resistant disease has become routine in patients with MHSPC. The CHAARTED and LATITUDE trials led to FDA approvals of docetaxel and abiraterone for $\mathrm{mHSPC}$ in 2015 and 2017 respectively; each demonstrating significant overall survival (OS) advantages over ADT alone[1, 2]. Enzalutamide and apalutamide approvals followed in 2019, based on positive data from ARCHES, ENZAMET, and TITAN trials [3-5].

Data to guide the optimal selection among these 1st line treatment regimens for $\mathrm{mHSPC}$ are limited. Meta-analyses comparing clinical trials of upfront docetaxel versus novel hormonal agents (NHA) in mHSPC have not shown any overall or progression-free survival benefit for either class of medication over the other [6-8]. To fill this gap, we performed a retrospective analysis of de novo mHSPC patients 
treated with docetaxel or novel hormonal agents (NHA) within the Mount Sinai Health System. We developed an automated oncology data retrieval and curation platform, and comprehensively extracted clinical features, outcomes, toxicities, and treatment patterns from the electronic medical record (EMR) in order to characterize and compare the clinical outcomes of patients treated with either upfront docetaxel or NHA for de novo mHSPC.

\section{Methods}

\section{Data Source}

Patients with prostate cancer were identified from the Mount Sinai Hospital at New York City data warehouse. Original EMR data from the hospital data warehouse were processed using the Sema4 Centrellis $^{\text {TM }}$ platform, consisting of automated abstraction engine, curation platform, patient dashboard, and cohort builder. Along with information from structured data fields, data from unstructured clinical notes were automatically abstracted, curated and integrated into structured fields. Cancer diagnosis metastases, medication, treatment, PSA values and other related data elements were stored in the database

\section{Patient Cohort Identification}

We identified prostate cancer patients diagnosed between January 2014 and April 2019 to capture the time frame when docetaxel and NHA were approved for mHSPC. 11,358 patients were assigned with at least one prostate cancer related ICD codes (185 in ICD-9 code and C61 in ICD-10 code) between January 1st, 2014 and April 30th, 2019 in the EMR system. However, not all patients with prostate cancer ICD codes assigned have confirmed prostate cancer. In addition, for patients diagnosed prior to visiting Mount Sinai hospital, their first time ICD code recorded in the EMR system didn't always match the date of diagnosis. To correctly identify patients with pathologically confirmed cancer diagnosis dates, we used the automated oncology data retrieval and curation platform developed by Sema4 as demonstrated in Supplemental Fig. 1A. Both structured information and extracted data elements from unstructured clinical note using heuristic rules were used to assign cancer diagnosis dates as demonstrated in Supplemental Fig. 1B. Patients with their extracted cancer diagnosis dates and other diagnosis related data elements, such as stage, histology, TNM, were classified into three groups using a stratification algorithm. Selected patients' data were manually reviewed by domain experts to evaluate the quality of the results We excluded patients diagnosed after April 2019 to ensure at least 12 months of follow-up. We defined de novo metastatic disease as clinical documentation of metastatic disease within 3 months of the diagnosis date. Upfront docetaxel and NHA use were determined by chart review. After the cohorts were identified, chart review was carried out to confirm duration of upfront docetaxel of NHA therapy, adverse events, identification of 2nd line therapy, and start date of 2nd line therapy. Additional clinical and demographic variables such as PSA values, age, sex, race, performance status, Gleason score, and metastasis burden at time of diagnosis were also evaluated. This study was approved by the Mount Sinai Institutional Review Board (Protocol number: STUDY-21-00442). 


\section{Failure-Free Survival Analysis}

Failure-free survival (FFS) was the primary outcome measure for this study, defined as time from initiation of either docetaxel or NHA to the next line of treatment. For the purposes of this metric, any change or addition of systemic therapy - including chemotherapy, NHA immunotherapy, and clinical trials - was considered a failure of therapy, regardless of the reason for the change (e.g. progression, toxicity, patient preference, physician discretion). Patients not known to receive a 2nd line treatment were censored at the time of their last follow-up. FFS was represented as Kaplan-Meier curves as detailed under Statistical Analysis.

\section{PSA Progression Free Survival (PFS) Analysis}

PSA PFS was defined as the time from initiation of therapy to first PSA increase $\geq 25 \%$ and $\geq 2 \mathrm{ng} / \mathrm{mL}$ above that patient's nadir, confirmed by a second value at least 3 weeks later, per PCWG3 criteria[9]. Patients who were started on 2nd line therapy before criteria for PSA progression were met were excluded from this analysis. Patients who did not have PSA progression and who had not started 2nd line therapy were censored at the time of their last follow-up. PSA PFS was represented as Kaplan-Meier curves as detailed under Statistical Analysis.

\section{Metastasis Burden and Failure Free Survival}

High metastasis burden was defined as visceral metastases or $\geq 4$ bone lesions with $\geq 1$ beyond the vertebral bodies and pelvis, per the CHAARTED trial[2]. Patients were assigned: high, low, or uncertain metastatic burden based on chart review by an oncologist. Differences in FFS between patient with high and low burden disease were compared in the 1. overall mHSPC cohort; 2. docetaxel treated cohort; and 3. NHA treated cohort. We further compared FFS between patients treated with docetaxel or NHA, stratified by metastasis burden. FFS were represented as Kaplan-Meier curves as detailed under Statistical Analysis.

\section{PSA nadir and Failure-Free Survival}

The post-treatment PSA in the docetaxel cohort was defined as the PSA value following completion of at least 4 cycles of docetaxel therapy. Patients who started 2nd line treatment prior to finishing 4 cycles of docetaxel (e.g. due to toxicity or refractory disease) were excluded from this analysis. For the NHA cohort, post-treatment PSA value was recorded after patients had received at least 7 months of ADT and at least 3 months of NHA. Patient were categorized in the docetaxel and NHA cohorts into three separate groups based on post-treatment PSA values: $\leq 0.2,0.2-4.0$, or $\geq 4.0 \mathrm{ng} / \mathrm{mL}$. We compared FFS according to posttreatment PSA within each treatment cohort.

\section{Statistical Analysis}

Failure-free survival and PSA progression free survival data were presented as Kaplan-Meier curves. Survival curves were created with R (version 3.5.0; http://www.R-project.org/.) using the survival package (version 2.44-1; https://cran.r-project.org/web/packages/survival/index.html) and report the log rank p- 
value according to default parameters. We examined potential confounding variables that may also be associated with clinical outcome using single variable and multivariable Cox proportional hazards regression, reporting the hazard ratios and the Wald test statistic $p$-value for each variable. PSA values were log transformed due to the heavy right tail, and 2 individuals with missing values were assigned to the median value.

\section{Results}

\section{Patient cohort}

Total 5725 patients were identified by the platform with pathologically confirmed diagnosis dates for prostate cancer between January 1, 2014 to April 30, 2019. Of these, 240 were determined to have had de novo metastatic disease, based on clinical documentation of metastatic disease within 3 months of the diagnosis date. The diagnosis date, metastatic status, and date of metastasis detection were based on terms extracted from clinical notes and pathology reports via the abstraction engine described above. We then identified patients who had been treated with docetaxel $(\mathrm{N}=67)$ or an NHA (abiraterone or enzalutamide) $(N=99)$ at any time. Finally, we identified those who had been treated with upfront docetaxel $(\mathrm{N}=52)$ or an NHA $(\mathrm{N}=42)$, defined as treatment within 7 months of diagnosis in conjunction with $A D T$ and in the absence of any prior systemic treatment for prostate cancer.

The two groups of de novo mHSPC patients were balanced with respect to baseline characteristics; there were no significant differences in age, race/ethnicity, PSA at diagnosis, Gleason score, ECOG performance status, and metastatic burden. Table 1 report the standardized mean difference (SMD) value. Variables with $\mathrm{SMD}<0.1$ are considered well matched between the two groups). The patient selection process is presented in Supplemental Fig. 2.

\section{Docetaxel treatment course}

In the docetaxel cohort, 47 of $52(90.4 \%)$ patients began treatment at the standard $75 \mathrm{mg} / \mathrm{m} 2$ dosing; 10 patients had subsequent dose reduction to $60 \mathrm{mg} / \mathrm{m} 2$. The remainder of patients, $5 / 52$ (9.6\%), started at a reduced dose of $60 \mathrm{mg} / \mathrm{m} 2 ; 1$ patient required a dose reduction to $45 \mathrm{mg} / \mathrm{m} 2$ (Supplemental Table 1A). The adverse effects leading to dose reduction in these 11 patients included: fatigue (3), neuropathy (3), febrile neutropenia (3), infection (1), and bradycardia (1) (Supplemental Table 1B).

Forty-five patients (86.5\%) completed 6 cycles of treatment. Six patients $(11.5 \%)$ terminated docetaxel early due to adverse effects, most commonly leg edema, fatigue, LFT abnormalities, anaphylactic infusion reaction. One patient (1.9\%) had docetaxel terminated before 6 cycles and moved to 2 nd line treatment due to refractory disease.

Forty-two docetaxel-treated patients (82.7\%) initiated subsequent therapy during the follow-up period. The most common subsequent therapy was an NHA such as abiraterone $(14 / 52,26.9 \%)$ or enzalutamide 
$(11 / 52,21.2 \%)$. Eight $(15.4 \%)$ patients enrolled in clinical trial following docetaxel therapy. Other agents included bicalutamide (3), sipuleucel-T (3), cabazitaxel (2), and apalutamide (2) (Supplemental Table 2).

\section{Novel hormonal agent (NHA) treatment course}

In the NHA cohort, 40 of 42 (95.2\%) patients received abiraterone, and 2 of $42(4.8 \%)$ received enzalutamide. All patients were started at each agent's respective approved dosing: abiraterone $1000 \mathrm{mg}$ daily with prednisone $5 \mathrm{mg}$ daily, or enzalutamide $160 \mathrm{mg}$ daily. Dose reductions occurred in 4 (9.5\%) patients, primarily due to abnormal LFTs (2) or hot flashes (1). (Supplemental Table 3)

Of the 42 patients treated with upfront NHA, $20(47.6 \%)$ patients transitioned to 2 nd line therapy during the follow-up period. $10 \%(2 / 20)$ of patients who moved onto 2 nd line therapy did so within first 3 months of 1 st line therapy due to rising PSA levels (Supplemental Table 4). The most common subsequent therapy following upfront NHA was an alternative NHA, e.g. enzalutamide $(13 / 20,65 \%)$. Three $(15 \%)$ patients received docetaxel, one patient each received radium-223 (5\%), olaparib (5\%), Sipuleucel-T (5\%) and bicalutamide (5\%) (Supplemental Table 4).

\section{Failure-free survival}

Patients who received upfront NHA had a significantly longer median FFS of 20.7 months $(95 \% \mathrm{Cl}=17.0$ $\mathrm{NE}$ [not estimable]) compared to 10.1 months $(95 \% \mathrm{Cl} 8.75-18.2)$ in docetaxel treated patients $(p=0.023)$ (Fig. 1A). Single and multivariable analyses are presented in Table 2. NHA, low metastasis burden, and lower baseline PSA levels are all associated with longer FFS. Variables with pvalues $<0.15$ were retained for the multivariable regression model. In the multi-variable model adjusting for age, baseline PSA, and metastasis burden, docetaxel was associated with worse FFS compared to NHA (HR 1.96, 95\% $\mathrm{Cl} 1.12$ $3.45, p=0.019$ ) (Table 2). None of the other variables remain statistically significant, but age below 65 at time of diagnosis, high baseline PSA, and high metastatic burden are still modestly associated with shorter FFS, irrespective of treatment received (Table 2,).

Clinical trials have suggested that high metastatic disease burden is predictive for clinical benefit from upfront treatment strategies for $\operatorname{mHSPC}[1,2,10]$. We sought to determine whether there was evidence for heterogeneous treatment effects based on disease burden. Patients with high metastasis burden had a significantly longer FFS with NHAs than docetaxel (25.12 [95\% Cl 17.03-NE] vs. 9.63 [95\% Cl 8.38-14.53] months, $p=0.014$ ) (Fig. 2A), while there was no significant difference in FFS among patients with low metastasis burden (NHA 20.71 [95\% Cl 12.92-NE] months vs. Docetaxel 26.5 [95\% Cl 8.65-NE] months, $p$ $=0.9)$ (Fig. 2B). However in a cox regression model predicting FFS, the interaction between upfront therapy and metastasis burden was not significant $(p=0.28)$

We further analyzed the prognostic implications of metastasis burden in the combined mHSPC cohort and in the individual docetaxel and NHA cohorts separately. In the combined cohort of docetaxel and $\mathrm{NHA}$ treated patients, a high metastatic burden of disease was associated with a shorter median FFS as compared to patients with low burden of disease, 11.57 [95\% Cl 9.24-20.12] vs. 20.71 [95\% Cl 12.92-NE] months $(p=0.034)$ (Fig. 2C). In the docetaxel group, high burden disease is associated with shorter 
median FFS as well, 9.63 [95\% Cl 8.38-14.53] vs. 26.5 [95\% Cl 8.65-NE] months ( $p=0.026)$ (Fig. 2D). However, metastasis burden was not associated with a significant FFS difference for patients that received NHA (25.12 [95\% Cl 17.03-NE] vs. 20.71 [95\% Cl 12.92-NE] months, $p=0.79$, Fig. 2E).

\section{PSA progression}

Since time to next treatment may be influenced by decisions to change therapy due to toxicity rather than disease progression, we also evaluated PSA progression between the two treatment groups. Of note, 7 of 52 docetaxel-treated patients were started on a subsequent line of therapy prior to completing 6 cycles of docetaxel treatment (due to toxicity [6 patients] or refractory disease [1 patient], as mentioned above); PSA progression after docetaxel alone could not be evaluated in these patients. Among evaluable patients, we found that those treated with docetaxel were more likely to start a subsequent therapy in the context of rising PSA levels prior to meeting PCWG criteria for PSA progression (14/45 vs. 4/42, $p=0.02$ ). The median PSA difference between PSA nadir and PSA at the start of 2nd treatment was 0.68 vs 1.58 $\mathrm{ng} / \mathrm{ml}$ among those treated with docetaxel vs NHAs $(p=0.55)$.

The co-variants were reasonably well matched between the remaining 30 docetaxel-treated patients and $38 \mathrm{NHA}$-treated patients with respect to age, race, log PSA at time of diagnosis, Gleason score, ECOG score, and metastasis burden (Supplemental Table 5). Among these patients, there was a trend towards longer PSA PFS with NHA compared to docetaxel, though the difference was not statistically significant (19.0 [95\% Cl 15.6-NE] vs. 13.2 months [95\% Cl 7.7-22.6], $p=0.069$ ) (Fig. 1B). Supplemental Table 6 presents the results for cox regression for both the univariable and multivariable analyses. In multivariable analysis, treatment (docetaxel vs. NHA) was not independently associated with PSA PFS (HR $1.64,95 \% \mathrm{Cl} 0.87-3.09 ; \mathrm{p}=0.13$ ) (Supplemental Table 6. Higher log PSA at time of diagnosis was independently associated with worse PSA PFS (Supplemental Table 6).

\section{Post-treatment PSA as Prognostic Marker in de novo mHSPC}

Prior to the advent of combination therapies for de novo mHSPC, when such patients were treated with ADT alone, post-ADT PSA levels were shown to be independent predictors of overall survival[11]. It is not known whether post-treatment PSA also holds prognostic value in the context of modern combination treatments.

To evaluate the prognostic significance of post-treatment PSA, we examined FFS stratified by posttreatment PSA in the overall study cohort, as well as in the individual docetaxel and NHA cohorts. Patients were stratified into 3 groups based on post-treatment PSA: $\leq 0.2,0.2-4$, and $\geq 4$. In the combined docetaxel and NHA cohorts evaluable for PSA response, patients with a post-treatment PSA $\leq 0.2$ had the longest FFS (58.95 [95\% Cl 20.71-NE] vs. 11.57 [ 95\% Cl 9.63-25.12] vs. 9.40 [95\% Cl 7.76-20.84] months, $p<0.001)$ (Fig. 3A). Similar outcomes were observed in the individual docetaxel and NHA cohorts (Fig. 3B and C)). 
In a multivariable analysis of the combined cohort, higher post-treatment PSA was independently associated with worse FFS (0.2-4 vs. $\leq 0.2 \mathrm{HR} 5.06,95 \% \mathrm{Cl} 1.98-12.97 ; \geq 4$ vs. $\leq 0.2 \mathrm{HR} 6.42,95 \% \mathrm{Cl}$ 2.43-16.95) (Table 3). In the NHA cohort, similar analysis demonstrated that the PSA 0.2-4 and PSA $\geq 4$ groups have significantly worse outcomes compared to the patients in the PSA $\leq 0.2$ group (HR $6.01,95 \%$ $\mathrm{Cl} 1.54-23.44$; HR 6.83, 95\% Cl 1.33-35.09) (Supplemental Table 7. For docetaxel, there is a trend towards worse outcomes among those with higher post-treatment PSA levels, $(0.2-4 \mathrm{vs}$. $\leq 0.2$ HR 3.42 , $95 \% \mathrm{Cl} 0.84-13.88, \geq 4$ vs. $\leq 0.2 \mathrm{HR} 4.35,95 \% \mathrm{Cl} 1.02-18.55)$ with the outcome between post-treatment PSA levels $\geq 4$ vs. $\leq 0.2$ being statistically significant $(p=0.046)$ (Supplemental Table 7).

\section{Discussion}

The treatment paradigm for $\mathrm{mHSPC}$ has rapidly shifted over the past 5 years from ADT monotherapy to combinations of ADT with docetaxel or NHAs such as abiraterone, enzalutamide, and apalutamide. However, data comparing these treatment options is limited. Network meta-analyses have addressed the question indirectly using cross-trial comparisons, finding no difference in overall survival between docetaxel and NHAs [6-8]. An opportunistic analysis from the STAMPEDE trial with limited power found improved failure-free survival and progression-free survival with abiraterone versus docetaxel, but no difference in overall survival [10].

Observational data may improve our understanding of the real-world use and outcomes of these treatments. We therefore conducted this study to compare the real-world use and outcomes of docetaxel vs. NHA in de novo mHSPC patients. To our knowledge, this is the first observational analysis to directly address this question.

\section{Failure Free Survival and Metastasis Burden}

Upfront NHA was associated with longer median FFS (measured by time to next treatment) than docetaxel and remained an independent predictor of FFS after controlling for demographic and clinical factors. This corresponds with findings from STAMPEDE, where the NHA cohort also showed a significantly longer FFS than the docetaxel cohort[10].

Time to next treatment was shorter with docetaxel than NHAs. Several factors may have contributed to this observation. First, more docetaxel-treated patients changed therapy due to toxicity ( 6 vs. $0, p=0.022)$. Second, there appeared to be a lower clinical threshold for docetaxel-treated patients to be initiated on a 2nd line therapy compared to NHA-treated patients. For example, 14 docetaxel-treated patients were started on a 2nd line therapy before meeting formal criteria for PSA progression, compared to only 4 of NHA-treated patients $(p=0.03)$. In addition, the median increase in PSA from nadir at the time of 2 nd line treatment initiation was $0.68 \mathrm{ng} / \mathrm{ml}$ among docetaxel-treated patients compared to $1.58 \mathrm{ng} / \mathrm{ml}$ among $\mathrm{NHA}$ treated patients; this difference was not statistically significant $(p=0.55)$, but suggests that clinicians were more inclined to initiate 2 nd line therapy in docetaxel-treated patients than NHA-treated 
patients. The fact that docetaxel treatment is limited to 6 cycles whereas NHAs are taken on an ongoing basis may influence clinicians' response to rising PSA levels.

High burden of metastatic disease has been associated with greater overall survival (OS) benefit from upfront docetaxel in post-hoc analyses of clinical trials. In our FFS analysis, NHAs were associated with median FFS of over 20 months regardless of disease burden (high: 25.12 vs. low: 20.71 months) while median FFS after docetaxel was significantly shorter in patients with high rather than low metastatic burden ( 9.63 vs. 26.5 months, $p=0.014$ ). These observations raise the possibility of treatment effect heterogeneity between docetaxel and NHAs by metastatic burden. We assessed the interaction term, which was not significant $(p=0.28)$, but this analysis was limited by sample size. We were unable to assess OS in this cohort due to a lack of events; thus while NHAs were associated with longer FFS in high-burden patients, it is not clear whether this would translate into an OS benefit.

\section{Second-Line Therapy}

At the end of follow-up, $17.3 \%$, of patients remained on ADT alone after completion of docetaxel, whereas $52.4 \%$ of the NHA cohort remained on 1 st line therapy. This was due in part to earlier approval for docetaxel in this setting and, consequently, longer follow-up in the docetaxel cohort (maximum of 64 months) compared to the NHA cohort (maximum of 28 months). The apparent lower clinical threshold to initiate 2 nd line treatment among docetaxel-treated patients, described above, may also have contributed.

Patterns of toxicity leading to dose reductions of treatment drug reflect otherwise well-described adverse effect profiles. Most patients, $86.5 \%$, were able to successfully complete 6 cycles of docetaxel, with the main reasons for early termination being fatigue, peripheral edema, and LFT abnormalities. Dose reductions in docetaxel were recommended for fatigue, neuropathy, and febrile neutropenia. Meanwhile, no clear cases of early termination of an NHA was observed, and dose reductions occurred only in a minority of patients, primarily in response to abnormal LFT's or hot flashes.

The prevailing practice pattern following upfront docetaxel was to transition to an NHA, with a relatively even split between abiraterone and enzalutamide. Following upfront NHA, most practitioners opted to transition to a second NHA. However, understanding that sequencing two different AR-pathway targeting agents back to back is usually associated with relatively low rates of response[12], more recent studies have explored transitioning to chemotherapy[13], radium, or targeted therapy with PARP inhibitor[14].

\section{PSA as a Prognostic Indicator}

In the pre-CHAARTED era, where standard of care for mHSPC was ADT alone, post-ADT induction PSA level was shown to have strong prognostic significance, with lower PSA levels associated with better survival outcomes[11]. To our knowledge, there is no data examining the prognostic value of posttreatment PSA in the context of modern combination therapies for mHSPC. In the overall study cohort, we observed that patients with a post-treatment PSA of $\leq 0.2$ enjoyed the longest FFS, whereas those with PSA 0.2-4 and $\geq 4$ were associated with significantly shorter FFS. This suggests that the post-treatment 
PSA after combination treatment in $\mathrm{MHSPC}$ patients has prognostic value, though further evaluation of its association with overall survival is necessary.

\section{Limitations}

There are inherent limitations in our data given the retrospective nature of this study. Comparisons between the docetaxel and NHA-treated patients may be confounded by indication. We were unable to perform propensity-score matching due to limited sample size. However, baseline characteristics were not significantly different between the two groups.

Enzalutamide and apalutamide, which were recently approved for $\mathrm{mHSPC}$, were not well-represented in our dataset. Future studies should address these newer agents as experience with them accrues.

We were unable to evaluate overall survival due to a limited number of events. We chose time to next treatment as a clinically relevant intermediate endpoint. Time to next treatment may be affected by several factors including frequency of follow-up, toxicity, and patient or provider-preference. We provided information on the decision to change treatment where available and found that most treatment changes were prompted by PSA progression. We conducted a separate PSA PFS analysis among a subset of evaluable patients which showed a trend towards longer PSA PFS with NHAs compared to docetaxel, though this association was not significant. Larger observational cohorts or prospective data are needed to compare long-term survival outcomes between these two treatment strategies for mHPSC.

\section{Conclusion}

In conclusion, this retrospective single-center study observed that upfront NHA treatment for de novo $\mathrm{mHSPC}$ was associated with longer FFS compared to docetaxel. Median FFS was longer among high-burden patients treated with NHAs rather than docetaxel, whereas median FFS was similar regardless of treatment in low-burden patients; the interaction was not significant. Finally, we showed that in the context of modern combination treatments for mHSPC, post-treatment PSA levels were associated with time to next treatment, suggesting prognostic value of this marker which should be evaluated in larger, prospective datasets.

\section{Abbreviations}

NHA-novel hormonal agents; mHSPC- metastatic hormone sensitive prostate cancer; ADT- androgen deprivation therapy; FFS- failure-free survival; EMR-electronic medical records; SDM- standardized mean difference; PFS- progression free survival; OS-overall survival

\section{Declarations}

\section{Ethics approval and consent to participate}


This retrospective analysis was approved by the Mount Sinai Health System Institutional Review Board (Protocol number: STUDY-21-00442). All experimental protocol for involving human data is in accordance with the Mount Sinai Health System (institutional) and Declaration of Helsinki guidelines. Participant consent was obtained in writing before they were included in the database for analysis and the study was approved by the Mount Sinai Health System ethics committee as mentioned above.

\section{Consent for publication}

Not applicable

\section{Availability of data and materials}

The datasets generated and analyzed during the current study are not publicly available as it is part of Sema 4 company database but are available from the corresponding author on reasonable request.

\section{Competing Interests}

The authors have declared no conflict of interest

\section{Funding}

None declared

\section{Author's Contribution}

WO, BL, RC conceived the project and advised. ES, XZ, TP, TM build the Sema4 database. KA, SG, PB, MD, MK, TO, MF analyzed the data. SG, TJ, BL, SN wrote the manuscript. All authors have read and approved the manuscript.

\section{Acknowledgements}

Non

\section{References}

1. Fizazi K, Tran N, Fein L, Matsubara N, Rodriguez-Antolin A, Alekseev BY, et al. Abiraterone plus Prednisone in Metastatic, Castration-Sensitive Prostate Cancer. N Engl J Med. 2017;377:352-60.

2. Sweeney CJ, Chen Y-H, Carducci M, Liu G, Jarrard DF, Eisenberger M, et al. Chemohormonal Therapy in Metastatic Hormone-Sensitive Prostate Cancer. N Engl J Med. 2015;373:737-46.

3. Armstrong AJ, Szmulewitz RZ, Petrylak DP, Holzbeierlein J, Villers A, Azad A, et al. ARCHES: A Randomized, Phase III Study of Androgen Deprivation Therapy With Enzalutamide or Placebo in Men With Metastatic Hormone-Sensitive Prostate Cancer. J Clin Oncol. 2019;37:2974-86. 
4. Chi KN, Agarwal N, Bjartell A, Chung BH, Pereira de Santana Gomes AJ, Given R, et al. Apalutamide for Metastatic, Castration-Sensitive Prostate Cancer. N Engl J Med. 2019;381:13-24.

5. Davis ID, Martin AJ, Stockler MR, Begbie S, Chi KN, Chowdhury S, et al. Enzalutamide with Standard First-Line Therapy in Metastatic Prostate Cancer. N Engl J Med. 2019;381:121-31.

6. Wallis CJD, Klaassen Z, Bhindi B, Goldberg H, Chandrasekar T, Farrell AM, et al. Comparison of Abiraterone Acetate and Docetaxel with Androgen Deprivation Therapy in High-risk and Metastatic Hormone-naïve Prostate Cancer: A Systematic Review and Network Meta-analysis. Eur Urol. 2018;73:834-44.

7. Feyerabend S, Saad F, Li T, Ito T, Diels J, Van Sanden S, et al. Survival benefit, disease progression and quality-of-life outcomes of abiraterone acetate plus prednisone versus docetaxel in metastatic hormonesensitive prostate cancer: A network meta-analysis. Eur J Cancer. 2018;103:78-87.

8. Vale CL, Fisher DJ, White IR, Carpenter JR, Burdett S, Clarke NW, et al. What is the optimal systemic treatment of men with metastatic, hormone-naive prostate cancer? A STOPCAP systematic review and network meta-analysis. Ann Oncol. 2018;29:1249-57.

9. Scher HI, Morris MJ, Stadler WM, Higano C, Basch E, Fizazi K, et al. Trial Design and Objectives for Castration-Resistant Prostate Cancer: Updated Recommendations From the Prostate Cancer Clinical Trials Working Group 3. J Clin Oncol. 2016;34:1402-18.

10. Sydes MR, Spears MR, Mason MD, Clarke NW, Dearnaley DP, de Bono JS, et al. Adding abiraterone or docetaxel to long-term hormone therapy for prostate cancer: directly randomised data from the STAMPEDE multi-arm, multi-stage platform protocol. Ann Oncol. 2018;29:1235-48.

11. Hussain M, Tangen CM, Higano C, Schelhammer PF, Faulkner J, Crawford ED, et al. Absolute prostatespecific antigen value after androgen deprivation is a strong independent predictor of survival in new metastatic prostate cancer: data from Southwest Oncology Group Trial 9346 (INT-0162). J Clin Oncol. 2006;24:3984-90.

12. Khalaf DJ, Annala M, Taavitsainen S, Finch DL, Oja C, Vergidis J, et al. Optimal sequencing of enzalutamide and abiraterone acetate plus prednisone in metastatic castration-resistant prostate cancer: a multicentre, randomised, open-label, phase 2, crossover trial. Lancet Oncol. 2019;20:1730-9.

13. de Wit R, de Bono J, Sternberg CN, Fizazi K, Tombal B, Wülfing C, et al. Cabazitaxel versus Abiraterone or Enzalutamide in Metastatic Prostate Cancer. N Engl J Med. 2019;381:2506-18.

14. de Bono J, Mateo J, Fizazi K, Saad F, Shore N, Sandhu S, et al. Olaparib for Metastatic CastrationResistant Prostate Cancer. N Engl J Med. 2020;382:2091-102.

\section{Tables}


Due to technical limitations, table 1-3 is only available as a download in the Supplemental Files section.

\section{Figures}

A

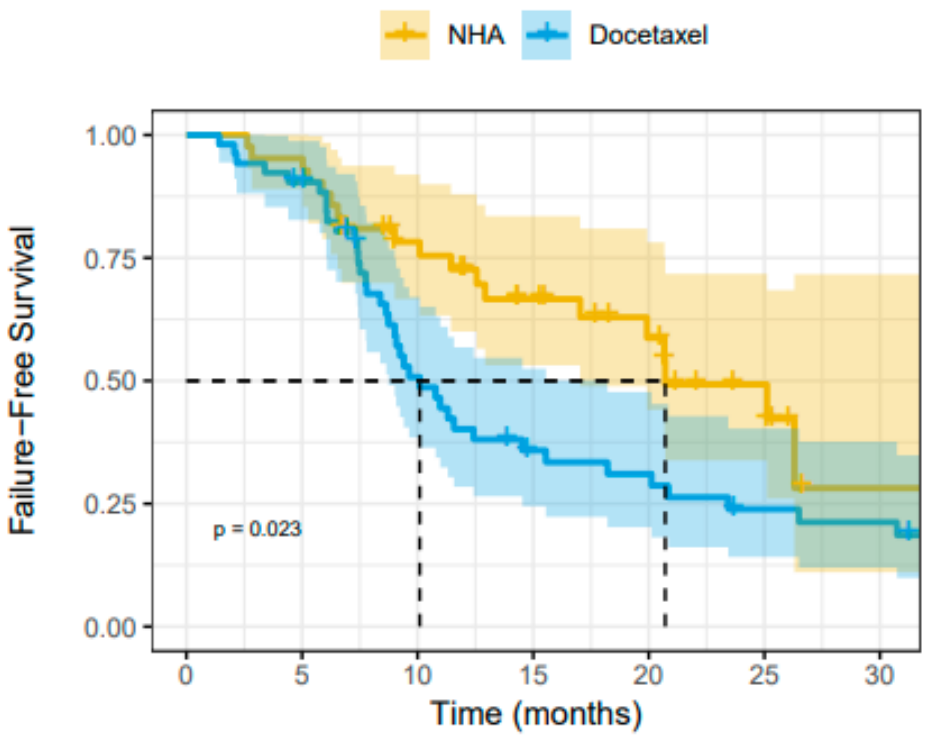

Number at risk (number censored)
B

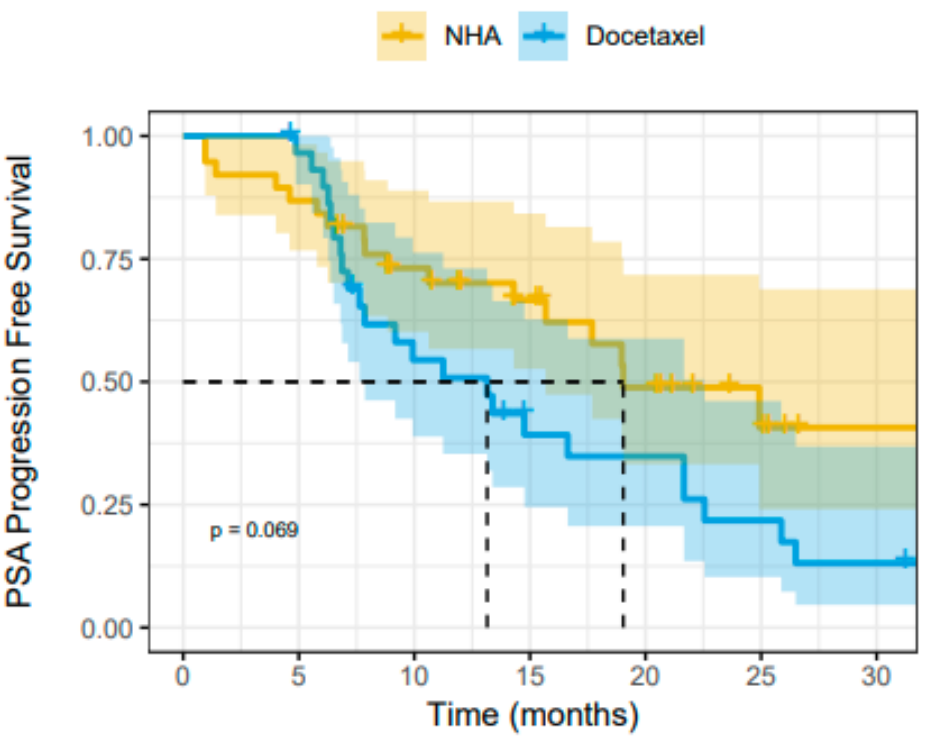

Number at risk (number censored)

\section{Figure 1}

Kaplan-Meier analysis comparing mHSPC patients treated with upfront docetaxel versus novel hormonal therapy (NHA) using A. Failure-free survival and B. PSA Progression free survival. 
A

$\perp$ NHA $\perp$ Docetaxel
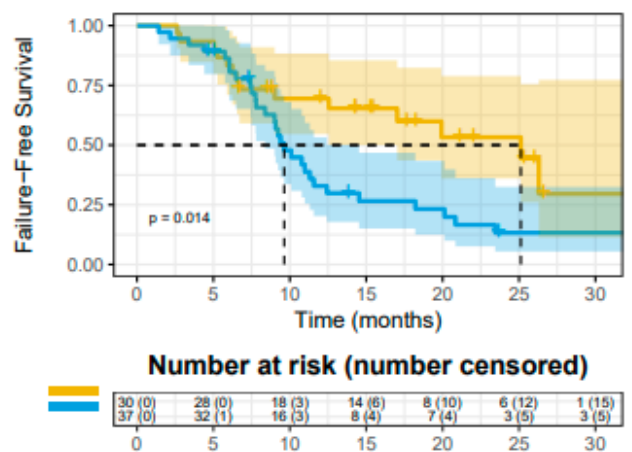

C

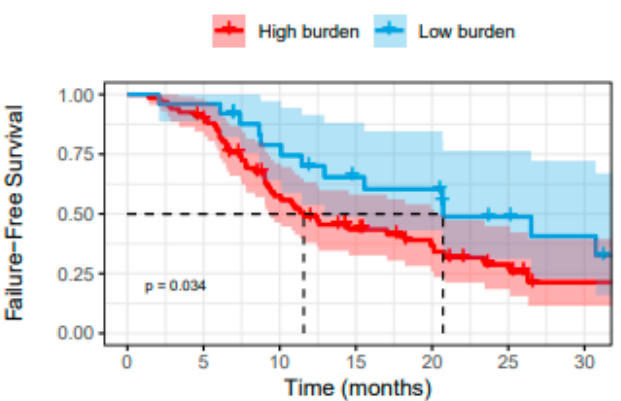

Number at risk (number censored)

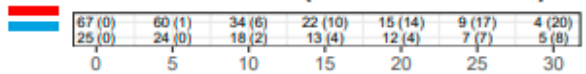

B
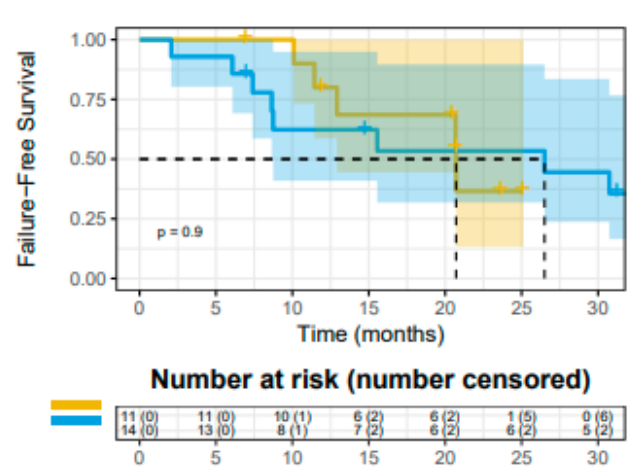

D

High burden + Low burden

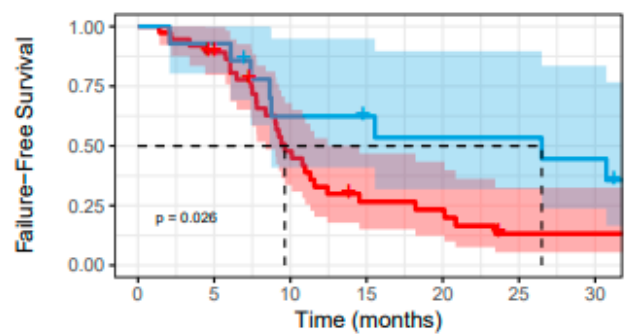

Number at risk (number censored)

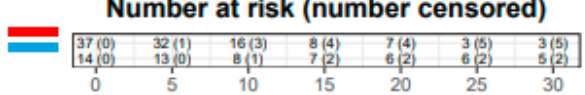

E

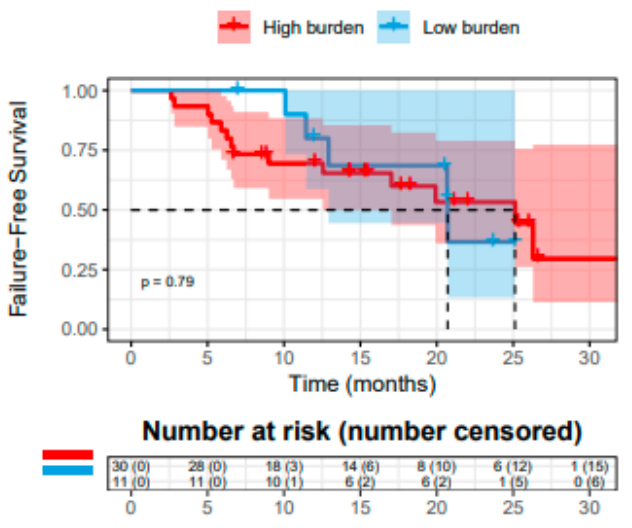

Figure 2

Kaplan-Meier analysis for FFS comparing patients treated with upfront docetaxel versus upfront NHA in A. High metastasis B. Low metastasis burden patients. Kaplan-Meier analysis for failure-free survival (FFS) comparing patients with high and low metastasis burden in C. all mHSPC patients, D. Patients treated with upfront docetaxel and E. Patients treated with upfront NHA.

A

+PSA $<0.2 \pm$ PSA $0.2-4 \neq$ PSA 4+
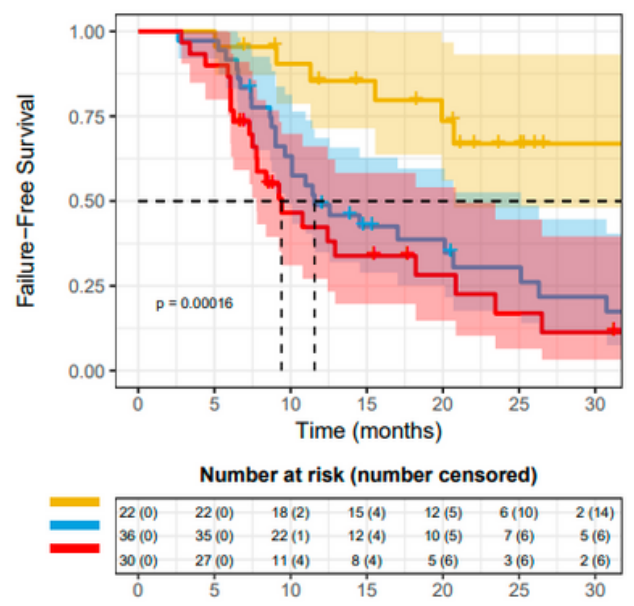

B

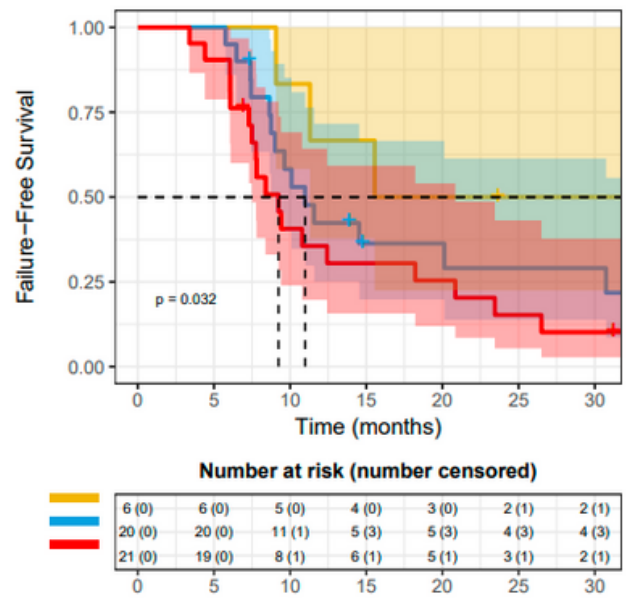

C

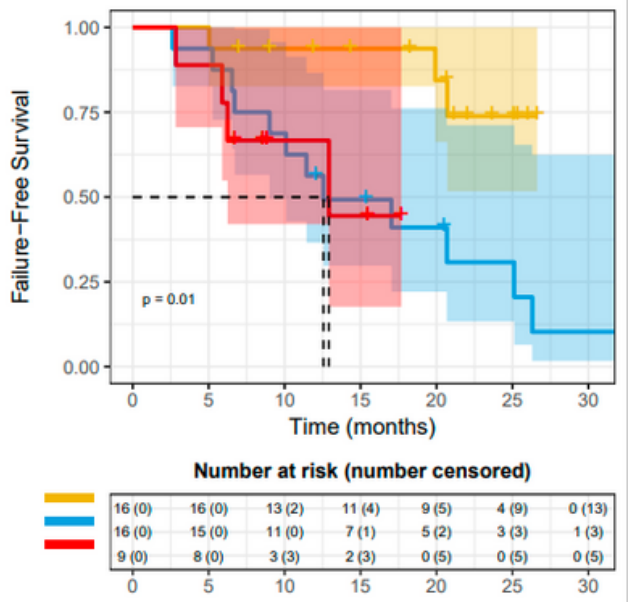

Figure 3 
Kaplan-Meier analysis for failure-free survival (FFS) comparing patients stratified by drop in PSA after treatment as mentioned in Methods in A. All mHSPC patients; B. upfront Docetaxel; C. upfront NHA treated patients.

\section{Supplementary Files}

This is a list of supplementary files associated with this preprint. Click to download.

- SupplementalFigure1.pptx

- SupplementalFigure2.pptx

- SupplementalFigureandTablelegends.docx

- SupplementalTable1.xlsx

- SupplementalTable2.xlsx

- SupplementalTable3.xlsx

- SupplementalTable4.xlsx

- SupplementalTable5.xlsx

- SupplementalTable6.xlsx

- SupplementalTable7.xIsx

- Table1.xlsx

- Table2.xlsx

- Table3.xlsx 